\title{
Comparative Study of Lipophilic Statin Activity in 2D and 3D in vitro Models of Human Breast Cancer Cell Lines MDA-MB-23I and MCF-7
}

This article was published in the following Dove Press journal: OncoTargets and Therapy

\section{Meda Bytautaite Vilma Petrikaite \\ Laboratory of Drug Targets Histopathology, Institute of Cardiology, Lithuanian University of Health Sciences, Kaunas, LT-50162, Lithuania}

Correspondence: Vilma Petrikaite Laboratory of Drug Targets Histopathology, Institute of Cardiology, Lithuanian University of Health Sciences, Sukilèlių Pr. 13, Kaunas LT-50I62, Lithuania

Tel +37068629383

Email vilmapetrikaite@gmail.com
Introduction: Statins are a type of drugs that are used to lower cholesterol level in blood. Since the early 1990s, it has been known that statins could be beneficial in cancer therapy. However, data remain controversial, especially regarding estrogen receptors status. Despite many studies in breast cancer models in vitro, the correlations of effects of separate statins in various model systems remain unclear.

Aim: Our aim was to evaluate the anticancer activity of lovastatin, mevastatin, pitavastatin and simvastatin on different subtypes of human breast cancer (MDA-MB-231 and MCF-7 cell lines) in spatially different 2D and 3D cultures in vitro.

Materials and Methods: Cell viability was tested using MTT assay. Effect of statins on cell colony formation was evaluated by calculating breast cancer cell colony area and number. The effect on cell migration was estimated by "wound healing" assay. The activity of compounds in $3 \mathrm{D}$ cultures was evaluated by measuring the spheroid size changes during incubation.

Results: Among the tested statins, pitavastatin had the greatest effect on the viability of breast cancer MDA-MB-231 and MCF-7 cell lines. The mevastatin and pitavastatin mostly decreased the MDA-MB-231 and MCF-7 cell colony formation. All statins at $90 \%$ of their estimated effective concentration $\left(\mathrm{EC}_{50}\right)$ and simvastatin at $10 \%$ of its $\mathrm{EC}_{50}$ concentration suppressed the MCF-7 cells migration in "wound healing" assay. Only higher concentrations of mevastatin and pitavastatin slowed down the MDA-MB-231 cell migration. Statins showed different activity on 3D cell cultures growth. Lovastatin and simvastatin delayed the growth of MDA-MB-231 cell spheroids, while mevastatin and pitavastatin reduced the growth of MCF-7 spheroids.

Conclusion: Statins possess different anticancer activity in human breast cancer MDA-MB -231 and MCF-7 cell cultures. Pitavastatin and simvastatin showed the highest activity in most tested assays, especially against MCF-7 cell line.

Keywords: statins, breast cancer, cell viability, cell migration, tumor spheroid, cell colonies

\section{Introduction}

Breast cancer is the most common cancer among women all over the world. ${ }^{1}$ Mastectomy and chemotherapy increase the survival of patients, but there always will be a limitation for the improvement of treatment without profound knowledge about the disease. ${ }^{2}$ Comprehensive analysis indicates that breast cancer subtypes have significant differences in pathway activity, progression, and response to medication. ${ }^{3}$ Breast cancer according to the status of estrogen receptor (ER), 
progesterone receptor (PR) and human epidermal growth factor receptor 2 (HER2) can be classified into Luminal A, Luminal B, HER2 positive and Basal-like subtypes. ${ }^{4}$ ER status is used as an indicator of endocrine responsiveness and as a prognostic factor for early recurrence in clinical trials. ER-positive tumors $(\mathrm{ER}+)$ compose most breast cancer cases, calculating for up to $75 \%$ of all cases especially in postmenopausal women. ${ }^{5}$

Statins are a type of drugs that are used to lower cholesterol level in blood. Their mechanism of action is related to the inhibition of an enzyme known as 3-hydroxy-3-methyglutaryl CoA (HMG-CoA) reductase. Statins have pleiotropic effects by inhibiting mevalonate pathway and having an impact on cell membrane integrity, signaling, protein synthesis, and cell cycle progression. ${ }^{6}$ Since the early 1990s, it has been known that statins could be successfully used in cancer therapy. The latest studies show that statins beside their cardiovascular effects exert desirable benefits for immunomodulation in autoimmune diseases and organ transplantation and prevent cancer growth. ${ }^{7}$ They show different effects depending on the cell line, statin concentration, duration of exposure of cells to statins and the type of statin being used. ${ }^{8,9}$ Furthermore, statins exert pro-apoptotic, anti-angiogenic, and immunomodulatory effects, which may inhibit cancer growth. Statins may prevent the growth of a variety of cancer cell types, including breast, gastric, pancreatic, and prostate carcinoma, neuroblastoma, melanoma, mesothelioma and acute myeloid leukemia cells. ${ }^{10}$ Cell incubation with statins induce the changes in cell cycle and apoptosis. After treatment of HMG-CoA inhibitors many apoptotic cell mechanisms are stimulated. ${ }^{11}$ In addition, statins used together with chemotherapeutic agents such as 5-fluorouracil, cisplatin and doxorubicin, increase their anticancer activity. ${ }^{12}$ Nonetheless, the more thorough analysis of statins' mechanism of action should be done to improve the survival of women. ${ }^{13}$

We decided to analyze the activity of four lipophilic statins: lovastatin, mevastatin, pitavastatin and simvastatin. Lipophilic statins have been shown to have anticancer activity, whereas the hydrophilic rosuvastatin and pravastatin have little or no effect on cancer cells. ${ }^{14,15}$ This phenomenon is explained by the fact that lipophilic statins cross cell membranes more easily and therefore enter tissues better. ${ }^{16}$ Hydrophilic statins have also been found to be more hepatoselective, and the lipophilic ones prefer to enter tissues other than the liver. ${ }^{17}$ This property of lipophilic statins could be favorable when they are used at high doses for a longer period that is usually required during the cancer treatment.

As a model system, we chose two different cancer subtypes: MCF-7 (Luminal A, ER+, HER2-) and very invasive, triple negative MDA-MB-231 (ER-, HER2-). ${ }^{18}$ Luminal A subtype is responsive to hormone therapy when basallike subtype is more aggressive and metastatic that makes it very difficult to treat. ${ }^{19}$ It is known that about $70 \%$ of all metastatic breast cancer patients have metastasis in bones and MDA-MB-231 cell line is more metastatic than MCF-7, therefore often used for research of breast cancer metastasis. ${ }^{20,21}$ In some earlier studies it was shown that cell lines which are ER- could be more sensitive to statins, ${ }^{13}$ but the preventive value of statins was observed in patients with ER+ tumors. ${ }^{22}$ However, data remain controversial, as in a recent clinical trial there was no association found between the used statin and ER status in tumor tissue. $^{23}$

We decided to test the effect of statins in 3D cocultures made from cancer cells and fibroblasts that better resemble tumor features in vivo. Using only $2 \mathrm{D}$ cultures often leads to false positive results that later turn out to be inefficient in the human body. ${ }^{24,25}$ Meanwhile, compound testing in cell 3D models reveals a more realistic drug response, captures phenotypic heterogeneity and better mimics the tumor microenvironment. Thus, we expected to reveal the differences of selected statin activity in human breast cancer cell lines of different subtypes and also to evaluate their effects in spatially different cancer 2D and 3D models in vitro. Furthermore, most experiments have been done when comparing the effects of several statins used at the same concentration, not taking too much attention to the variable compound effect on cell viability. In our studies, we decided to use statin concentrations at the same level from their half-maximal effective values $\left(\mathrm{EC}_{50}\right)$, in order to find their effects by excluding the factor of cell viability reduction.

\section{Materials and Methods \\ Materials}

Lovastatin and mevastatin were purchased from Alfa Aesar (Ward Hill, MA, USA). Pitavastatin calcium and simvastatin were bought from Abcam (Cambridge, UK).

\section{Cell Cultures}

The human breast cancer MDA-MB-231 and MCF-7 cell lines were obtained from the American Type Culture 
Collection (ATCC, Manassas, VA, USA). Human foreskin fibroblasts (HF) CRL-4001 were originally obtained from ATCC and kindly provided by Prof. Helder Santos (University of Helsinki, Finland). MDA-MB-231, MCF-7 and HF were cultured in Dulbecco's Modified Eagle's GlutaMAX medium (Gibco (Carlsbad, CA, USA)). Medium was supplemented with $10,000 \mathrm{U} / \mathrm{mL}$ penicillin, $10 \mathrm{mg} / \mathrm{mL}$ streptomycin (Gibco), and 10\% fetal bovine serum (Gibco). Cells were incubated at $37^{\circ} \mathrm{C}$ in a humidified atmosphere containing $5 \% \mathrm{CO}_{2}$. They were used until the passage of 20 .

\section{Cell Viability}

Cell viability was tested using 3-(4,5-dimethylthiazol-2-yl)2,5-diphenyltetrazolium bromide (MTT; Sigma-Aldrich Co., St Louis, MO, USA) assay, as described elsewhere. ${ }^{26}$ Briefly, MDA-MB-231 and MCF-7 cells were seeded in 96-well plates (Corning) in a volume of $100 \mu \mathrm{L}\left(5 \times 10^{3}\right.$ cells/well). After 24 hours preincubation, the cells were treated with 100 $\mu \mathrm{L}$ of various concentrations of statins. Only medium without cells was used as a positive control, and the medium with $0.5 \%$ DMSO (Sigma-Aldrich Co.) served as a negative control. After 72 hours the cells were incubated with the $0.5 \mathrm{mg} /$ $\mathrm{mL}$ of MTT solution (Sigma-Aldrich Co.) for the next 4 hours. The colored formazan product was dissolved in 100 $\mu$ L DMSO (Sigma-Aldrich Co.). The absorbance was measured spectrophotometrically at wavelengths of 570 and 630 $\mathrm{nm} . \mathrm{EC}_{50}$ values that represent the concentration of a compound causing $50 \%$ reduction of cancer cell metabolic activity has been calculated using the Hill equation.

\section{Cell Colony Formation}

MDA-MB-231 and MCF-7 cells were seeded into 12-well plates (Corning) at a density of 100 cells/well in $2 \mathrm{~mL}$ of medium and treated with of statin concentrations representing $10 \%$ and $90 \%$ of calculated $\mathrm{EC}_{50}$ value. Cells were incubated in a humidified atmosphere containing $5 \% \mathrm{CO}_{2}$ at $37^{\circ} \mathrm{C}$ and grown for 12 days. After incubation, the cells were rinsed with phosphate-buffered saline (PBS, Gibco) and fixed with 4\% paraformaldehyde (Thermo Fisher Scientific, Waltham, MA, USA) solution for 20 minutes. Then the cells were rinsed with PBS two more times, stained with $0.1 \%$ aqueous crystal violet solution (SigmaAldrich Co.) for 15 minutes, followed by rinses with sterile deionized water to remove the excess dye. Photos of colonies were taken using $G: B O X$ gel documentation system (Syngene International Ltd, Bengaluru, India) and Genesys software (Syngene International Ltd). The colony numbers and percentage area were calculated by GeneTools software (Syngene International Ltd). Cell colony area and number in control groups was normalized to $100 \%$. The percentage of drug-treated colony area and number were compared to the control.

\section{Cell Migration}

MDA-MB-231 and MCF-7 cells were seeded into 24-well plates (Corning) at a density of 500,000 cells/well in 500 $\mu \mathrm{L}$ of medium and incubated in a humidified atmosphere containing $5 \% \mathrm{CO}_{2}$ at $37^{\circ} \mathrm{C}$ for 1 day. Then the monolayers were scratched with a sterile $100 \mu \mathrm{L}$ pipette plastic tip in the center of the well. The old medium was changed for the fresh one containing statin concentrations representing $10 \%$ and $90 \%$ of calculated $\mathrm{EC}_{50}$ values. Images of "wounds" were taken every 24 hours for 3 days and the effect was evaluated by measuring the size of the "wound" area using ImageJ software (National Institutes of Health).

\section{Spheroid Growth}

Spheroids were formed from MDA-MB-231 and MCF-7 cells and fibroblast (1:1) by $3 D$ Bioprinting method. The cells were magnetized by adding nanoparticles NanoShuttle (Nano3D Biosciences Inc., Houston, TX, USA) for 8-10 hours. Once magnetized, the cells were resuspended and seeded into ultralow attachment 96-well plates (Corning) in a volume of 100 $\mu \mathrm{L}$ (2,000 breast cancer cells and 2,000 human fibroblasts per well). The plates were placed on a magnetic drive and incubated in a humidified atmosphere containing $5 \% \mathrm{CO}_{2}$ at $37^{\circ} \mathrm{C}$ until spheroids were formed. After 2 days of incubation, the photos of spheroids were taken using phase-contrast microscopy, and the medium was replaced by the fresh medium containing $10 \%$ and $90 \% \mathrm{EC}_{50}$ of statin solutions. Photos were taken every 48 hours and the medium was replaced every 96 hours. The effect of statins in 3D breast cancer cell cultures was determined by measuring the size change of spheroids using ImageJ software.

\section{Statistical Analysis}

All experiments were repeated at least three times, calculating the mean and standard deviation. The data was processed using Microsoft Office Excel 2016 software (Microsoft Corporation, Redmond, WA, USA) and IBM SPSS Statistics version 26.0 package. The level of statistical significance was set at $p<0.05$. In order to determine significant differences between values, analysis of variance (ANOVA) followed by a Tukey post-hoc test was performed. 


\section{Results and Discussion}

\section{Statins Reduce the Viability of Breast Cancer Cells}

All tested statins showed from 3 to 11 times greater antiproliferative effect on MDA-MB-231 than MCF-7 cell line (Figure 1). According to Litzenburger, the different breast cancer subtypes require the different treatment. MDA-MB -231 is an ER-negative subtype which is mostly affected by cell growth and progression inhibitors such as statins. ${ }^{27}$ Mueck et $\mathrm{al}^{28}$ also found that MDA-MB-231 cell viability is affected by lower concentrations of lipophilic statins than the MCF-7 cell line. ${ }^{28}$ However, the obtained $\mathrm{EC}_{50}$ values were much lower than those against normal human fibroblasts, ${ }^{29,30}$ that could allow us to prove again that statins are more selective towards cancer cells than the normal ones.

The most effective compound in both cell lines after 72 hours was pitavastatin $\left(\mathrm{EC}_{50}\right.$ values in MDA-MB-231 and MCF-7 cell lines were $0.24 \pm 0.04$ and $2.23 \pm 0.12 \mu \mathrm{M}$, respectively). The obtained results can be explained by the ability of statins to inhibit the enzyme HMG-CoA reductase: pitavastatin has the highest inhibitory activity, simvastatin, lovastatin has a slightly lower activity, and mevastatin has the weakest effect. ${ }^{31,32}$ Istvan also found that type II statins, which include pitavastatin, form more bonds with HMG-CoA reductase, which enhances their inhibitory effect compared to type I statins, which include the other compounds tested,

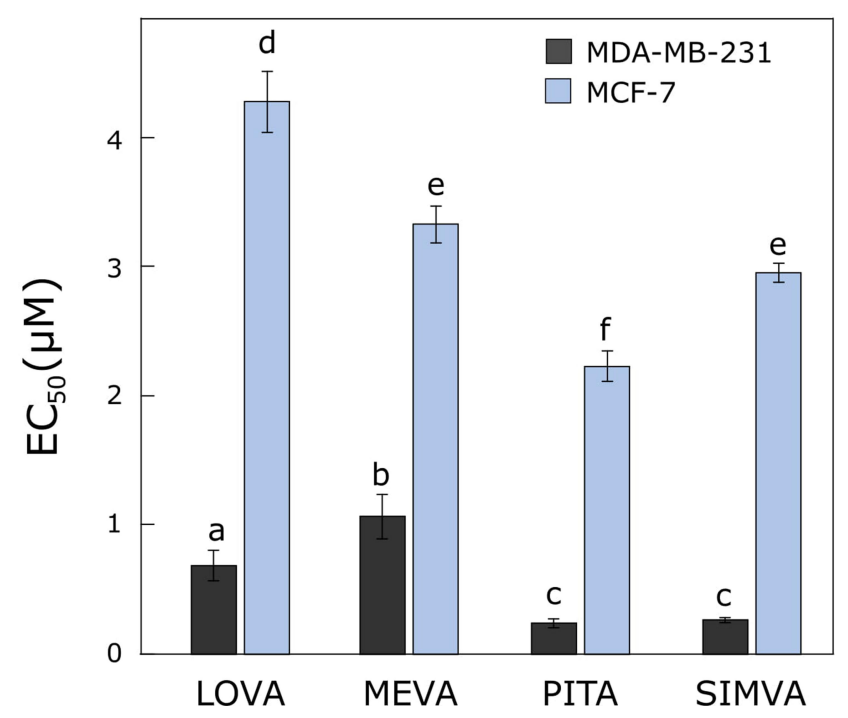

Figure I Activity of statins on cell viability. The $\mathrm{EC}_{50}$ values of lovastatin, mevastatin, pitavastatin and simvastatin after 72 hours in breast cancer MDA-MB-23I and MCF-7 cell lines. Bars marked with different letters indicate statistically significant differences $(p<0.05)$ within the same category.

Abbreviations: $\mathrm{EC}_{50}$, half maximal effective concentration; LOVA, lovastatin; MEVA, mevastatin; PITA, pitavastatin; SIMVA, simvastatin. mevastatin, lovastatin and simvastatin. ${ }^{33}$ Saito et al ${ }^{34}$ assumed that because of its unique structure (heptenoate as the basic structure, quinoline ring at the core and side chain has fluorophenyl and cyclopropyl moieties) pitavastatin could show an optimal activity as a HMG-CoA reductase inhibitor. ${ }^{34}$ Moreover, Paškevičiūte et $\mathrm{al}^{35}$ found that pitavastatin also had the highest effect among other statins in pancreatic cancer cell lines. ${ }^{35}$

For further research, we chose to use the concentrations of compounds equal to $10 \%$ and $90 \%$ from their calculated $\mathrm{EC}_{50}$ values, in order to avoid the possible misinterpretation of other effects in clonogenic and migration assays due to different possible effects on cell viability.

\section{Statins Suppress the Breast Cancer Cell Colony Formation}

MCF-7 colonies grew bigger than MDA-MB-231 after 12 days of incubation with tested statins (Figure 2A). All compounds reduced the MDA-MB-231 and MCF-7 number of colonies (Figure 2B). Furthermore, all the statins statistically significantly decreased the area of colonies (except $10 \%$ of $\mathrm{EC}_{50}$ lovastatin in the case MDA-MB-231 cell line) (Figure 2B).

Higher concentrations ( $90 \%$ of $\mathrm{EC}_{50}$ values) of mevastatin and pitavastatin showed the greatest effect on reducing the MDA-MB-231 and MCF-7 cell colony formation. Kanugula,${ }^{36}$ in his experiments used $5 \mu \mathrm{M}$ concentration fluvastatin, which is a synthetic compound like pitavastatin. $\mathrm{He}$ found out that this compound reduced the number of MDAMB-231 colonies four times more compared to the control. ${ }^{36}$ In addition, Cheng et $\mathrm{al}^{37}$ determined that $2.5 \mu \mathrm{M}$ fluvastatin reduced the number of MCF-7 colonies up to $25 \%{ }^{37}$ Moreover, Jung et $\mathrm{al}^{38}$ used $0.1 \mu \mathrm{M}$ concentration simvastatin in both MDA-MB-231 and MCF-7 cell lines. This compound was 3 times more effective on MDA-MB-231 colony formation. ${ }^{38}$ Buranrat et $a{ }^{39}$ found that simvastatin reduced MCF-7 colony formation by increasing compound concentrations from 2.5 to $50 \mu \mathrm{M}$, respectively. ${ }^{39}$ The results we obtained show that increasing the concentration of all tested statins increases their effect on breast cancer cell colony formation. However, except for pitavastatin, no significant big differences between different tested statins was observed in both cell lines.

\section{Statins Reduce the Migration of Breast Cancer Cells}

The lower concentration $\left(10 \%\right.$ of $\mathrm{EC}_{50}$ values $)$ of the tested compounds (except simvastatin in MCF-7 cell 


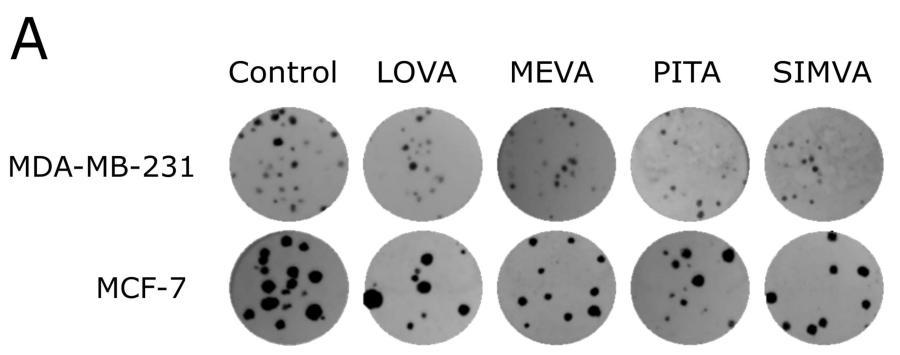

B

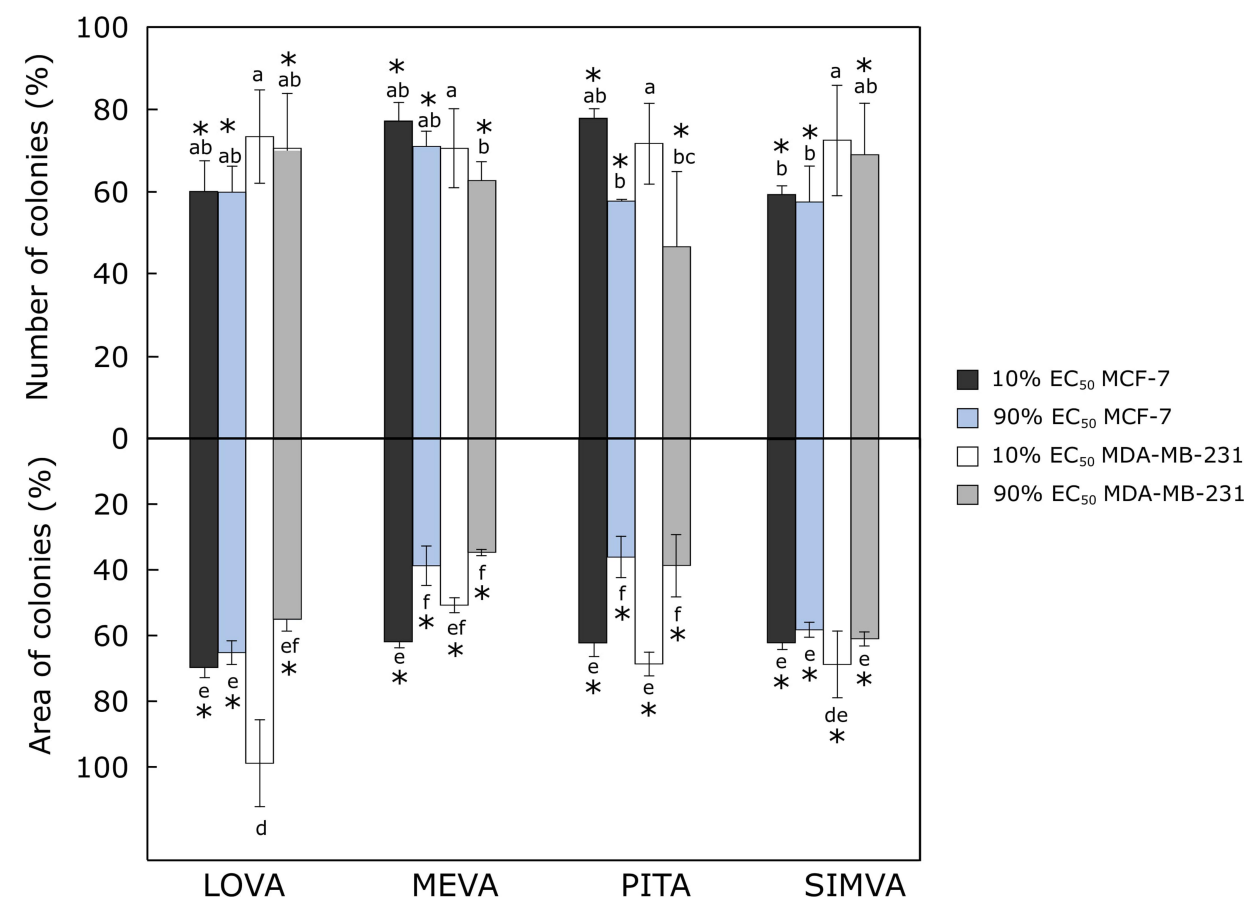

Figure 2 Effect of statins on colony formation. (A) Photos of cell colonies formed after 12 days of treatment with $90 \%$ of EC 50 values of statins. (B) Average number of colonies treated with the compounds and the percentage of area covered by cancer cell colonies. The asterisks $(*)$ indicate $p<0.05$ compared to control. Bars marked with different letters indicate statistically significant differences $(p<0.05)$ within the same category.

Abbreviations: $\mathrm{EC}_{50}$, half maximal effective concentration; LOVA, lovastatin; MEVA, mevastatin; PITA, pitavastatin; SIMVA, simvastatin.

line) did not have a significant effect on breast cancer cell migration. Furthermore, MDA-MB-231 cells was more migrant and also less affected by statins than MCF-7 cells. Gordon et $\mathrm{al}^{40}$ found out that MDA-MB-231 is a more invasive cell line than MCF-7, and it depends on the expression of ER and adhesion molecules. ${ }^{40}$

All higher concentrations ( $90 \%$ of $\mathrm{EC}_{50}$ values) of compounds, especially pitavastatin, inhibited MCF-7 cell migration (the size of the "wound" reached up to $36.19 \pm$ $6.85 \%$ ) (Figure 3A). Only higher concentrations of mevastatin and pitavastatin decreased the migration of MDAMB-231 cells (the size of the "wound" area was about $20 \%$ bigger than in control group). "Wounds" treated by lovastatin and simvastatin fully closed after 72 hours (Figure 3B). Mandal et $\mathrm{al}^{41}$ used $5 \mu \mathrm{M}$ concentration of simvastatin in MDA-MB-231, which was higher than our $0.27 \mu \mathrm{M}$ concentration, and successfully inhibited the migration of the cells after 12 hours. $^{41}$

Interestingly, despite statins being up to five times more effective in MTT assay against MDA-MB-231 cells compared to MCF-7 cell line, in the "wound healing" assay the results were totally opposite. As we used relatively normalized concentrations to the $\mathrm{EC}_{50}$ values, we could expect to avoid the compound influence on cell viability and see only the effect on cell migration. All tested statins at higher concentrations possessed a very similar effect on MCF-7 but not MDA-MB-231 cell migration. Pitavastatin and mevastatin significantly inhibited cell migration to the "wound" area compared to others, thus we could hypothesize that the anti-migrastatic 

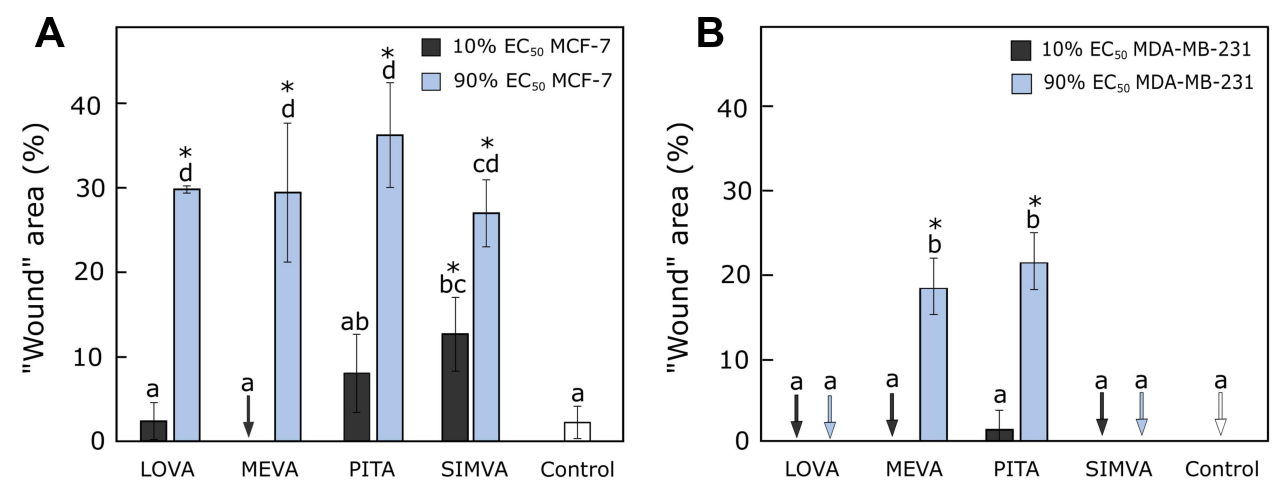

Figure 3 Activity of statins on cell migration. (A) The percentage of "wound" area after 72 hours treatment with statins in MCF-7 cell line. (B) The percentage of "wound" area after 72 hours treatment with statins in MDA-MB-23I cell line. Arrows mark the "wounds" that "closed" completely. The asterisks $(*)$ indicate $p<0.05$ compared to control. Bars marked with different letters indicate statistically significant differences $(p<0.05)$ within the same category.

Abbreviations: $\mathrm{EC}_{50}$, half maximal effective concentration; LOVA, lovastatin; MEVA, mevastatin; PITA, pitavastatin; SIMVA, simvastatin.

effect of these two compounds is related to some other mechanisms.

Several researchers have also suggested that statins could take a role in cancer cell migration and invasion by preventing the cholesterol synthesis. ${ }^{42}$ It is known that cholesterol takes part in cancer progression and some cancer cell types have a higher content of cholesterol in their membranes. ${ }^{43}$ Statins also can inhibit the steroid synthesis and thus reduce the risk of breast and other types of cancer that are related to the elevated concentrations of oxysterol. ${ }^{44,45}$

\section{Statins Effect on 3D Cultures (Spheroids)}

Nowadays 3D cultures are widely used to study the effect of new compounds. ${ }^{46}$ In our research breast cancer cells were mixed with fibroblasts to better represent the tumor microenvironment in vivo. Fibroblasts play a major role in tumor development, progression, angiogenesis, induction of metastasis, and suppression of the immune response. ${ }^{47}$

MDA-MB-231 spheroids formed compact 3D structures in 2 days (Figure 4A). MCF-7 spheroids were bigger, had uneven edges and grew only after 4 days (Figure 4B).

Statins showed different activity on $3 \mathrm{D}$ cell cultures growth. The higher used concentration of lovastatin and simvastatin delayed the growth of MDA-MB-231 cell spheroids (Figure 4C), while mevastatin and pitavastatin at higher concentrations reduced the growth of MCF-7 spheroids (Figure 4D). Other researchers also established that the same dose of statin has a bigger effect on MDA-MB-231 than MCF-7 3D cultures. ${ }^{48}$ The MCF-7 spheroids incubated with lower concentrations ( $10 \%$ of their $\mathrm{EC}_{50}$ values) of lovastatin and mevastatin, became even looser compared to the control group. It could mean that the higher concentration

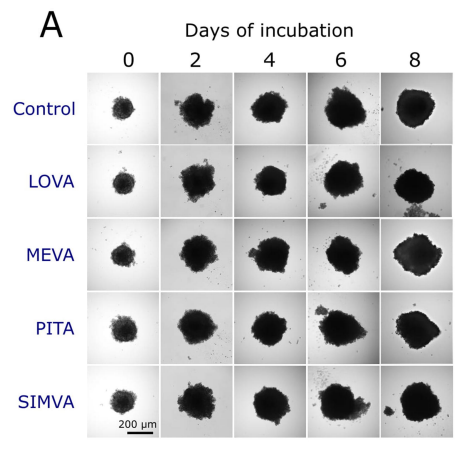

B Days of incubation
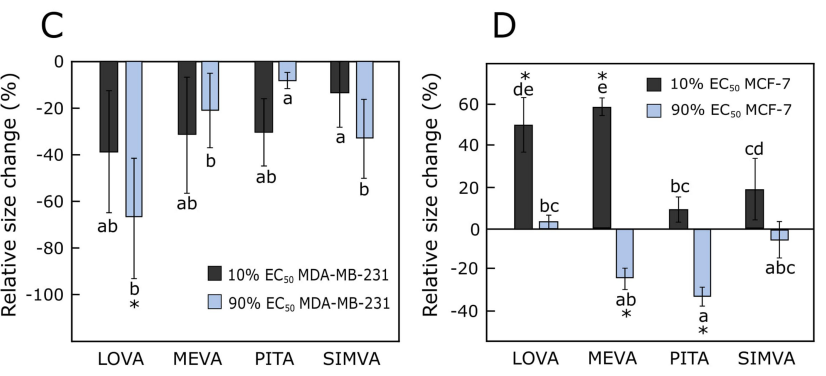

Figure 4 Effect of statins on 3D cultures. (A) MDA-MB-23I spheroids at day 0 and 8 of experiment, with higher concentration of statins. (B) MCF-7 spheroids at day 0 and 4 of experiment, with higher concentration of statins. (C) The relative size change of MDA-MB-23I spheroids compared to the control group after 10 days incubation with statins. (D) The relative size change of MCF-7 spheroids compared to the control group after 6 days incubation with statins. The asterisks $\left(^{*}\right)$ indicate $p<0.05$ compared to control. Bars marked with different letters indicate statistically significant differences $(p<0.05)$ within the same category.

Abbreviations: $\mathrm{EC}_{50}$, half maximal effective concentration; LOVA, lovastatin; MEVA, mevastatin; PITA, pitavastatin; SIMVA, simvastatin.

is related more to the cytotoxic effect of statins and spheroids become smaller, and relatively lower concentration of compounds produce the effects more related to cell-cell adhesion. Interestingly, all tested statins did not contribute to the "spheroid disintegrating" effect in the case of MDA-MB -231 spheroids. We hypothesize that possibly different concentrations of statins induce different mechanisms of actions in these two cell lines. 
In our study, we found out the different effect of statins in $2 \mathrm{D}$ and $3 \mathrm{D}$ breast cancer cultures. As expected, the compounds were more active in cell monolayers than in spheroids. Higher concentrations ( $90 \%$ of $\mathrm{EC}_{50}$ values) of statins only up to $10-20 \%$ reduced the actual spheroid size at the end of the experiment (after 6 and 10 days of incubation with compounds), and in the case of MCF-7 spheroids in some groups they became even bigger (Figure 4D).

There are some evidences, that statins do not produce the same effect in different cancer cell types. Due to their participation in the farnesylation process, the inactive forms of Ras, Rac and Rho proteins are found inside the cells. ${ }^{49}$ Highly invasive cell line MDA-MB-231, which is characterised by overexpression of RhoA and constitutive activation of Ras, was shown to be much more sensitive to cerivastatin treatment compared to the MCF-7 cell line (when not over-expressing RhoA). Also, similar correlations were estimated in some other cell lines. ${ }^{50}$ Another correlation was found between the level of NF- $\mathrm{KB}$ and sensitivity to statins in different types of breast cancer cell lines: MDA-MB-231 (the highest level), SKBR3 (moderate level), and MCF-7 (the lowest level). ${ }^{14}$ It could mean that statins or their derivatives could find their niche in cancer therapeutics, especially in more aggressive tumor types or in combinations with marketed anticancer drugs in order to prevent metastasis.

\section{Conclusion and Future Directions}

There is still a need to clarify whether statins could be beneficial in oncology. Some studies have reported a reduction in cancer-related mortality among statin users, but there are many controversies. In our research, statins possessed different anticancer activity in human breast cancer MDA-MB-231 and MCF-7 cell cultures in 2D assays (cell viability, clonogenic and "wound healing" assays) Pitavastatin and simvastatin showed the highest activity in most tested assays, especially against MCF-7 cell lines. Despite a much greater effect in the MCF-7 cell monolayer, all statins did not show much higher activity in MCF-7 spheroids compared to MDA-MB-231 3D cultures. Moreover, their effect in tumor spheroids was different between each other and also varied at different concentrations. Also, despite statins being more active in the MDAMB-231 cell line in the viability assay, they produced a higher anti-migrastatic effect in MCF-7 cell line. Mevastatin and pitavastatin more strongly inhibited MDAMB-231 cell migration compared to lovastatin and simvastatin, though their activity in 3D cultures was not high. The effect that contributes to spheroid disintegration and migration could be studied more thoroughly and it could contribute to a more detailed description of statin mechanism of action.

\section{Ethical Approval}

This study was approved by the Bioethical Center of Lithuanian University of Health Sciences prior to the research on cell lines.

\section{Author Contributions}

All authors made a significant contribution to the work reported, whether that was in the conception, study design, execution, acquisition of data, analysis and interpretation, or in all these areas; took part in drafting, revising or critically reviewing the article; gave final approval of the version to be published; have agreed on the journal to which the article has been submitted; and agree to be accountable for all aspects of the work.

\section{Funding}

This research did not receive any additional funding.

\section{Disclosure}

The authors report no conflicts of interest in this work.

\section{References}

1. WHO. Breast Cancer. https://www.who.int/cancer/prevention/diagno sis-screening/breast-cancer/en/. Accessed March 8, 2020.

2. Feng Y, Spezia M, Huang S, et al. Breast cancer development and progression: risk factors, cancer stem cells, signaling pathways, genomics, and molecular pathogenesis. Genes Dis. 2018;5(2):77-106. doi:10.1016/j.gendis.2018.05.001

3. Heiser LM, Sadanandam A, Kuo W-L, et al. Subtype and pathway specific responses to anticancer compounds in breast cancer. Proc Natl Acad Sci. 2012;109(8):2724-2729. doi:10.1073/pnas.1018854108

4. Dai X, Xiang L, Li T, Bai Z. Cancer Hallmarks Biomarkers and breast cancer molecular subtypes. $J$ Cancer. 2016;7(10):1281-1294. doi: $10.7150 /$ jca. 13141

5. Shomaf M, Masad J, Najjar S, Faydi D. Distribution of breast cancer subtypes among Jordanian women and correlation with histopathological grade: molecular subclassification study. JRSM Short Rep. doi: $10.1177 / 2042533313490516$

6. Demierre M-F, Higgins PDR, Gruber SB, Hawk E, Lippman SM. Statins and cancer prevention. Nat Rev Cancer. 2005;5(12):930-942. doi: $10.1038 / \mathrm{nrc} 1751$

7. Wyhe RDV, Rahal OM, Woodward WA. Effect of statins on breast cancer recurrence and mortality: a review. Breast Cancer. doi:10.2147/ BCTT.S148080

8. Matusewicz L, Meissner J, Toporkiewicz M, Sikorski AF. The effect of statins on cancer cells-review. Tumor Biol. 2015;36(7):4889-4904. doi:10.1007/s13277-015-3551-7

9. Abdullah MI, de Wolf E, Jawad MJ, Richardson A. The poor design of clinical trials of statins in oncology may explain their failure - lessons for drug repurposing. Cancer Treat Rev. 2018;69:84-89. doi:10.1016/j. ctrv.2018.06.010 
10. Vallianou NG, Kostantinou A, Kougias M, Kazazis C. Statins and cancer. Anticancer Agents Med Chem. 2014;14(5):706-712. doi:10.2174/1871520613666131129105035

11. Gopalan A, Yu W, Sanders BG, Kline K. Simvastatin inhibition of mevalonate pathway induces apoptosis in human breast cancer cells via activation of JNK/CHOP/DR5 signaling pathway. Cancer Lett. 2013;329(1):9-16. doi:10.1016/j.canlet.2012.08.031

12. Chan KKW, Oza AM, Siu LL. The statins as anticancer agents. Clin Cancer Res. 2003;9(1):10-19.

13. Kimbung S, Lettiero B, Feldt M, Bosch A, Borgquist S. High expression of cholesterol biosynthesis genes is associated with resistance to statin treatment and inferior survival in breast cancer. Oncotarget. 2016;7(37):59640-59651. doi:10.18632/oncotarget.10746

14. Campbell MJ, Esserman LJ, Zhou Y, et al. Breast cancer growth prevention by statins. Cancer Res. 2006;66(17):8707-8714. doi:10.1158/0008-5472.CAN-05-4061

15. Brown M, Hart C, Tawadros T, et al. The differential effects of statins on the metastatic behaviour of prostate cancer. Br J Cancer. 2012;106 (10):1689-1696. doi:10.1038/bjc.2012.138

16. Kato S, Smalley S, Sadarangani A, et al. Lipophilic but not hydrophilic statins selectively induce cell death in gynaecological cancers expressing high levels of HMGCoA reductase. J Cell Mol Med. 2010;14(5):1180-1193. doi:10.1111/j.1582-4934.2009.00771.x

17. Hamelin BA, Turgeon J. Hydrophilicity/lipophilicity: relevance for the pharmacology and clinical effects of $\mathrm{HMG}-\mathrm{CoA}$ reductase inhibitors. Trends Pharmacol Sci. 1998;19(1):26-37. doi:10.1016/ s0165-6147(97)01147-4

18. Dai X, Cheng H, Bai Z, Li J. Breast cancer cell line classification and its relevance with breast tumor subtyping. J Cancer. 2017;8 (16):3131-3141. doi:10.7150/jca.18457

19. Holliday DL, Speirs V. Choosing the right cell line for breast cancer research. Breast Cancer Res. 2011;13(4):215. doi:10.1186/ bcr2889

20. Pulido C, Vendrell I, Ferreira AR, et al. Bone metastasis risk factors in breast cancer. Ecancermedicalscience. 2017;11:715. doi:10.3332/ ecancer.2017.715

21. Xuan H, Li Z, Yan H, et al. Antitumor activity of chinese propolis in human breast cancer MCF-7 and MDA-MB-231 Cells. Evid Based Complement Alternat Med. 2014;2014:1-11. doi:10.1155/2014/ 280120

22. Bjarnadottir O, Feldt $M$, Inasu $M$, et al. Statin use, HMGCR expression, and breast cancer survival - the Malmö Diet and Cancer Study. Sci Rep. 2020;10(1):558. doi:10.1038/s41598-01957323-9

23. Borgquist S, Giobbie-Hurder A, Ahern TP, et al. Cholesterol, cholesterol-lowering medication use, and breast cancer outcome in the BIG 1-98 study. J Clin Oncol. 2017;35(11):1179-1188. doi:10.1200/JCO.2016.70.3116

24. Magnetic 3D cell culture in high throughput screening. PromoCell. August. 27. Available from: https://www.promocell.com/cells-in-action /magnetic-cell-culture-high-throughput-screening/. Accessed August 21, 2020.

25. 5 reasons cancer researchers adopt $3 \mathrm{~d}$ cell culture: a review of recent literature. Sigma-Aldrich. Available from: https://www.sigmaaldrich. com/technical-documents/articles/biology/5-reasons-cancer-researchers -adopt-3d-cell-culture-white-paper.html.Accessed June 10, 2020.

26. Grigalius I, Petrikaite V. Relationship between antioxidant and anticancer activity of trihydroxyflavones. Molecules. 2017;22:2169. doi:10.3390/molecules22122169

27. Litzenburger BC, Brown PH. Advances in preventive therapy for estrogen-receptor-negative breast cancer. Curr Breast Cancer Rep. 2014;6(2):96-109. doi:10.1007/s12609-014-0144-1

28. Mueck AO, Seeger H, Wallwiener D. Effect of statins combined with estradiol on the proliferation of human receptor-positive and receptor-negative breast cancer cells. Menopause. 2003;10(4):332. doi:10.1097/01.GME.0000055485.06076.00
29. Chen J-B, Chern T-R, Wei -T-T, Chen -C-C, Lin J-H, Fang J-M. Design and synthesis of dual-action inhibitors targeting histone deacetylases and 3-hydroxy-3-methylglutaryl coenzyme A reductase for cancer treatment. J Med Chem. 2013;56(9):3645-3655. doi:10.1021/ jm400179b

30. Wang L, Wang Y, Chen A, et al. Pitavastatin slows tumor progression and alters urine-derived volatile organic compounds through the mevalonate pathway. FASEB J. 2019;33(12):13710-13721. doi:10.1096/fj.201901388R

31. Igel M, Sudhop T, von Bergmann K. Pharmacology of 3-hydroxy3-methylglutaryl-coenzyme A reductase inhibitors (statins), including rosuvastatin and pitavastatin. J Clin Pharmacol. 2002;42(8):835-845. doi:10.1177/009127002401102731

32. Istvan ES, Deisenhofer J. Structural mechanism for statin inhibition of HMG-CoA reductase. Science. 2001;292(5519):1160-1164. doi: $10.1126 /$ science. 1059344

33. Istvan E. Statin inhibition of HMG-CoA reductase: a 3-dimensional view. Atheroscler Suppl. 2003;4(1):3-8. doi:10.1016/s1567-5688(03) 00003-5

34. Saito Y. Critical appraisal of the role of pitavastatin in treating dyslipidemias and achieving lipid goals. Vasc Health Risk Manag. 2009;5:921-936. doi:10.2147/VHRM.S5551

35. Paškevičiūtė M, Petrikaitė V. Differences of statin activity in 2D and 3D pancreatic cancer cell cultures. Drug Des Devel Ther. 2017;11:3273-3280. doi:10.2147/DDDT.S149411

36. Kanugula AK, Gollavilli PN, Vasamsetti SB, et al. Statin-induced inhibition of breast cancer proliferation and invasion involves attenuation of iron transport: intermediacy of nitric oxide and antioxidant defence mechanisms. FEBS J. 2014;281(16):3719-3738. doi:10.1111/febs. 12893

37. Cheng G, Lopez M, Zielonka J, et al. Mitochondria-targeted nitroxides exacerbate fluvastatin-mediated cytostatic and cytotoxic effects in breast cancer cells. Cancer Biol Ther. 2011;12(8):707-717. doi:10.4161/cbt.12.8.16441

38. Jung HH, Lee S-H, Kim J-Y, Ahn JS, Park YH, Im Y-H. Statins affect ETS1 -overexpressing triple-negative breast cancer cells by restoring DUSP4 deficiency. Sci Rep. 2016;6(1):33035. doi:10.1038/srep33035

39. Buranrat B, Suwannaloet W, Naowaboot J. Simvastatin potentiates doxorubicin activity against MCF-7 breast cancer cells. Oncol Lett. 2017;14(5):6243-6250. doi:10.3892/ol.2017.6783

40. Gordon LA, Mulligan KT, Maxwell-Jones H, Adams M, Walker RA, Jones JL. Breast cell invasive potential relates to the myoepithelial phenotype. Int J Cancer. 2003;106(1):8-16. doi:10.1002/ijc.11172

41. Mandal CC, Ghosh-Choudhury N, Yoneda T, Choudhury GG, GhoshChoudhury N. Simvastatin prevents skeletal metastasis of breast cancer by an antagonistic interplay between p53 and CD44. J Biol Chem. 2011;286(13):11314-11327. doi:10.1074/jbc.M110.193714

42. Denoyelle C, Vasse M, Körner M, et al. Cerivastatin, an inhibitor of HMG-CoA reductase, inhibits the signaling pathways involved in the invasiveness and metastatic properties of highly invasive breast cancer cell lines: an in vitro study. Carcinogenesis. 2001;22 (8):1139-1148. doi:10.1093/carcin/22.8.1139

43. Chimento A, Casaburi I, Avena P, et al. Cholesterol and its metabolites in tumor growth: therapeutic potential of statins in cancer treatment. Front Endocrinol. 2019;9. doi:10.3389/fendo.2018.00807

44. Raza S, Meyer M, Goodyear C, Hammer KDP, Guo B, Ghribi O. The cholesterol metabolite 27-hydroxycholesterol stimulates cell proliferation via ER $\beta$ in prostate cancer cells. Cancer Cell Int. 2017;17:52. doi:10.1186/s12935-017-0422-x

45. Wu Q, Ishikawa T, Sirianni R, et al. 27-hydroxycholesterol promotes cell-autonomous, ER-positive breast cancer growth. Cell Rep. 2013;5 (3):637-645. doi:10.1016/j.celrep.2013.10.006

46. Edmondson R, Broglie JJ, Adcock AF, Yang L. Three-dimensional cell culture systems and their applications in drug discovery and cell-based biosensors. Assay Drug Dev Technol. 2014;12 (4):207-218. doi:10.1089/adt.2014.573 
47. Zoetemelk M, Rausch M, Colin DJ, Dormond O, Nowak-Sliwinska P. Short-term 3D culture systems of various complexity for treatment optimization of colorectal carcinoma. Sci Rep. 2019;9(1):7103. doi:10.1038/s41598-019-42836-0

48. Lovitt CJ, Shelper TB, Avery VM. Miniaturized three-dimensional cancer model for drug evaluation. Assay Drug Dev Technol. 2013;11 (7):435-448. doi:10.1089/adt.2012.483

49. Zhu Y, Casey PJ, Kumar AP, Pervaiz S. Deciphering the signaling networks underlying simvastatin-induced apoptosis in human cancer cells: evidence for non-canonical activation of RhoA and Rac1 GTPases. Cell Death Dis. 2013;4(4):e568-e568. doi:10.1038/cddis.2013.103
50. Matusewicz L, Meissner J, Toporkiewicz M, Sikorski AF. The effect of statins on cancer cells-review. Tumour Biol J Int Soc Oncodevelopmental Biol Med. 2015;36(7):4889-4904. doi:10.1007/ s13277-015-3551-7

\section{Publish your work in this journal}

OncoTargets and Therapy is an international, peer-reviewed, open access journal focusing on the pathological basis of all cancers, potential targets for therapy and treatment protocols employed to improve the management of cancer patients. The journal also focuses on the impact of management programs and new therapeutic

Submit your manuscript here: https://www.dovepress.com/oncotargets-and-therapy-journa agents and protocols on patient perspectives such as quality of life, adherence and satisfaction. The manuscript management system is completely online and includes a very quick and fair peer-review system, which is all easy to use. Visit http://www.dovepress.com/ testimonials.php to read real quotes from published authors. 